\title{
A NOTE ON COMMUTATIVITY OF UNBOUNDED REPRESENTATIONS
}

\author{
SCHÔICHI ÔTA
}

(Communicated by Palle E. T. Jorgensen)

\begin{abstract}
Commutativity for unbounded representations is studied in terms of the Cayley transform.
\end{abstract}

\section{INTRODUCTION AND NOTATION}

We study commutativity of unbounded representations of a $*$-algebra. In our previous paper [5], we introduced the notion of strong commutativity for representations by using the strong commutants of representations and gave some results related to an extension of a representation. In this note we study the relation between the strong commutativity of representations and their corresponding Cayley transforms. Moreover, in relation to a question on the integrable extension of a representation, we show that there is no integrable extension of a representation under a pointwise commutativity condition on the corresponding Cayley transforms.

Let $\mathfrak{A}$ be a $*$-algebra with unit $e$ and $\mathfrak{A}_{h}$ be the set of all hermitian elements of $\mathfrak{A}$. Let $\pi$ be a linear mapping of $\mathfrak{A}$ into all closable linear operators on a common dense subspace $\mathscr{D}(\pi)$ of a Hilbert space $\mathscr{H}$ such that $\mathscr{D}(\pi)$ is invariant under each $\pi(x) \quad(x \in \mathfrak{A})$. If $\pi$ satisfies that $\pi(e)=I$, the identity operator on $\mathscr{H}$, and $\pi(x) \pi(y) \eta=\pi(x y) \eta$ for all $x, y \in \mathfrak{A}$ and $\eta \in \mathscr{D}(\pi)$, and also $\pi$ satisfies $(\pi(x) \xi, \eta)=\left(\xi, \pi\left(x^{*}\right) \eta\right)$ for all $x \in \mathfrak{A}$ and $\xi, \eta \in \mathscr{D}(\pi)$, then $\pi$ is said to be a $*$-representation of $\mathscr{A}$ on $\mathscr{H}$. If the domain $\mathscr{D}(\pi)$ is complete with respect to the induced topology given by the family of seminorms $\{\|\pi(x) \xi\|: x \in \mathfrak{A}\}, \pi$ is called closed. The adjoint representation $\pi^{*}$ is defined by

$$
\begin{aligned}
& \mathscr{D}\left(\pi^{*}\right)=\bigcap_{x \in \mathfrak{A}} \mathscr{D}\left(\pi(x)^{*}\right), \\
& \left.\pi^{*}(x)=\left.\pi\left(x^{*}\right)^{*}\right|_{\mathscr{D}\left(\pi^{*}\right)} \quad \text { (the restriction of } \pi\left(x^{*}\right)^{*} \text { to } \mathscr{D}\left(\pi^{*}\right)\right) .
\end{aligned}
$$

If $\pi=\pi^{*}$, we call $\pi$ selfadjoint. A closed $*$-representation $\pi$ is called integrable (or standard in terms of [6]) if $\overline{\pi\left(a^{*}\right)}=\pi(a)^{*}$ for all $a \in \mathfrak{A}$, where the

Received by the editors August 26, 1991 and, in revised form, October 1, 1991.

1980 Mathematics Subject Classification (1985 Revision). Primary 47D40, 46K40.

Key words and phrases. Unbounded representation, strong commutativity, Cayley transformation, extension. 
bar denotes the closure of an operator. If $\pi$ is integrable, then it is selfadjoint. We note that a closed *-representation $\pi$ is integrable if and only if $\pi(a)$ is essentially selfadjoint for all $a \in \mathfrak{A}_{h}$.

For a bounded operator $B$ and a densely defined operator $K$ in $\mathscr{H}$, we say that $B$ commutes with $K$ if the relation $B K \subseteq K B$ holds; namely, $B$ leaves the domain $\mathscr{D}(K)$ invariant and $B K \xi=K B \xi$ for all $\xi \in \mathscr{D}(K)$. Also we say that $B$ weakly commutes with $K$ if $B K \subseteq K^{*} B$ holds. We next recall the definitions of the strong and weak commutants of a representation. For a representation $\pi$ of $\mathfrak{A}$ on $\mathscr{H}$, the weak commutant $\mathscr{C}^{w}(\pi)$ is defined by

$$
\mathscr{C}^{w}(\pi)=\left\{T \in \mathscr{B}(\mathscr{H}): T \pi(x) \subseteq \pi^{*}(x) T \text { for all } x \in \mathfrak{A}\right\},
$$

and the strong commutant $\mathscr{C}^{s}(\pi)$ is defined by

$$
\mathscr{C}^{s}(\pi)=\{T \in \mathscr{B}(\mathscr{H}): T \pi(x) \subseteq \pi(x) T \text { for all } x \in \mathfrak{A}\} .
$$

Here $\mathscr{B}(\mathscr{H})$ denotes the algebra of all bounded linear operators on $\mathscr{H}$. Then $\mathscr{C}^{w}(\pi)$ is a weakly closed set of $\mathscr{B}(\mathscr{H})$ and is $*$-closed; that is, it is closed under the usual adjoint operation of $\mathscr{B}(\mathscr{H})$, but it need not be an algebra. On the other hand, $\mathscr{C}^{s}(\pi)$ is an algebra, but it is not *-closed in general. If $\pi$ is selfadjoint, then $\mathscr{C}^{s}(\pi)$ is a von Neumann algebra with $\mathscr{C}^{s}(\pi)=\mathscr{C}^{w}(\pi)$. For further details on unbounded representations, we refer to [7].

\section{Strong commutativity and CAYLEy transform}

Let $\pi_{1}$ and $\pi_{2}$ be $*$-representations of $\mathfrak{A}$ on $\mathscr{H}$. We say [5] that $\pi_{1}$ strongly commutes with $\pi_{2}$ if the commutant of $\mathscr{C}^{s}\left(\pi_{1}\right)$ is contained in $\mathscr{C}^{s}\left(\pi_{2}\right)$; $\mathscr{C}^{s}\left(\pi_{1}\right)^{\prime} \subseteq \mathscr{C}^{s}\left(\pi_{2}\right)$. Here, for a subset $\mathscr{M}$ of $\mathscr{B}(\mathscr{H}), \mathscr{M}^{\prime}$ denotes the usual commutant of $\mathscr{M}$.

For a $*$-representation $\pi$ of $\mathfrak{A}$ on $\mathscr{H}$ and $x \in \mathfrak{A}_{h}$, let $V_{\pi(x)}$ be the Cayley transform of the closed symmetric operator $\overline{\pi(x)}$;

$$
V_{\pi(x)}=(\overline{\pi(x)}-i)(\overline{\pi(x)}+i)^{-1},
$$

where $i=\sqrt{-1}$. Then, as usual, $V_{\pi(x)}$ is extended to the partial isometry on $\mathscr{H}$ that is zero on the deficiency space $\operatorname{ker}\left(\pi(x)^{*}-i\right) \equiv \mathscr{R}(\overline{\pi(x)}+i)^{\perp}$, the orthogonal complement of the range of $\overline{\pi(x)}+i$, and it is also denoted by $V_{\pi(x)}$.

Lemma 1. For each $x \in \mathfrak{A}_{h}, V_{\pi(x)}^{*}=V_{\pi(-x)}$.

Proof. It is easy to see that $V_{\pi(x)}^{*}=(-\overline{\pi(x)}-i)(-\overline{\pi(x)}+i)^{-1}$ on $\mathscr{R}(-\overline{\pi(x)}+i)$, the range of $-\overline{\pi(x)}+i$. On the other hand, $V_{\pi(x)}=0$ on $\mathscr{R}(\overline{\pi(x)}+i)^{\perp}$ by the definition. It follows that $V_{\pi(x)}^{*}=0$ on $\mathscr{R}(-\overline{\pi(x)}+i)^{\perp}$. Clearly, $-\overline{\pi(x)}=$ $\overline{\pi(-x)}$. Hence, we have $V_{\pi(x)}^{*}=V_{\pi(-x)}$.

Proposition 2. Let $\pi$ be a *-representation of $\mathfrak{A}$. If $\mathscr{C}^{s}(\pi)$ is *-closed, then $V_{\pi(x)}$ belongs to $\mathscr{C}^{s}(\pi)^{\prime}$ for all $x \in \mathfrak{A}_{h}$.

Proof. Let $x$ be in $\mathfrak{A}_{h}$ and take any $T$ in $\mathscr{C}^{s}(\pi)$. Since $T$ commutes with $\overline{\pi(x)}$, it follows that

$$
T V_{\pi(x)}=V_{\pi(x)} T \text { on } \mathscr{R}(\overline{\pi(x)}+i) .
$$


On the other hand, since $T^{*}$ also belongs to $\mathscr{C}^{s}(\pi)$ by our assumption, $T \pi(x)^{*}$ $\subseteq \pi(x)^{*} T$. Therefore, for $\xi \in \mathscr{D}\left(\pi(x)^{*}\right)$ with $\pi(x)^{*} \xi=i \xi$, we have $T \xi \in$ $\mathscr{D}\left(\pi(x)^{*}\right)$ and $\pi(x)^{*} T \xi=T \pi(x)^{*} \xi=i T \xi$. Thus $T V_{\pi(x)}=V_{\pi(x)} T=0$ on $\operatorname{ker}\left(\pi(x)^{*}-i\right)$, which implies the proposition.

In order to discuss the relation between the strong commutativity of representations and the Cayley transforms corresponding to hermitian elements of $\mathfrak{A}$, we consider the following condition $(\star)$ for $*$-representations $\pi$ and $\rho$ of $\mathfrak{A}$ :

$$
V_{\pi(x)} V_{\rho(y)}=V_{\rho(y)} V_{\pi(x)} \quad \text { for all } x, y \in \mathfrak{A}_{h} .
$$

Proposition 3. Suppose $\pi$ and $\rho$ are *-representations of $\mathfrak{A}$ such that

$$
\left\{V_{\pi(x)}: x \in \mathfrak{A}_{h}\right\} \subseteq \mathscr{C}^{s}(\rho) ;
$$

then the condition $(\star)$ is satisfied.

Proof. For each $x \in \mathfrak{A}_{h}$, by Lemma 1 and the condition $(\star \star)$, both $V_{\pi(x)}$ and $V_{\pi(x)}^{*}$ belong to $\mathscr{C}^{s}(\rho)$ for all $x \in \mathfrak{A}_{h}$. In the proof of Proposition 2, replace $\pi$ by $\rho$ and $T$ by $V_{\pi(x)}$, respectively. Then the proposition follows from the same arguments as in the proof.

The following corollary is a direct consequence of Propositions 2 and 3.

Corollary 4. If $\pi$ strongly commutes with $\rho$ and $\mathscr{C}^{s}(\pi)$ is *-closed, then the condition $(\star)$ is satisfied.

Conversely, we give the following:

Theorem 5. Let $\pi$ and $\rho$ be *-representation of $\mathfrak{A}$. If $\pi$ and $\rho$ satisfy the condition $(\star)$, then

$$
\left\{V_{\pi(x)}: x \in \mathfrak{A}_{h}\right\} \subseteq \mathscr{C}^{w}(\rho) \text { and }\left\{V_{\rho(x)}: x \in \mathfrak{A}_{h}\right\} \subseteq \mathscr{C}^{w}(\pi)
$$

Proof. Let $x$ be in $\mathfrak{A}_{h}$. Then we have only to show $V_{\pi(x)} \in \mathscr{C}^{w}(\rho)$. Since $V_{\pi(x)}$ commutes with $V_{\rho(y)}$ for all $y \in \mathfrak{A}_{h}$, it follows that

$$
V_{\pi(x)}\left(I-V_{\rho(y)}\right)^{-1} \subseteq\left(I-V_{\rho(y)}\right)^{-1} V_{\pi(x)} .
$$

Since $\overline{\rho(y)} \subseteq i\left(I+V_{\rho(y)}\right)\left(I-V_{\rho(y)}\right)^{-1} \subseteq \rho(y)^{*}$, we have

$$
\begin{aligned}
V_{\pi(x)} \overline{\rho(y)} & \subseteq i\left(I+V_{\rho(y)}\right) V_{\pi(x)}\left(I-V_{\rho(y)}\right)^{-1} \\
& \subseteq i\left(I+V_{\rho(y)}\right)\left(I-V_{\rho(y)}\right)^{-1} V_{\pi(x)} \subseteq \rho(y)^{*} V_{\pi(x)} .
\end{aligned}
$$

It follows from [7, Corollary 8.2.8] that $V_{\pi(x)} \in \mathscr{C}^{w}(\rho)$. This completes the proof.

Theorem 6. Let $\pi$ be an integrable representation of $\mathfrak{A}$, and let $\rho$ be a *representation of $\mathfrak{A}$ with $\mathscr{C}^{s}(\rho)=\mathscr{C}^{w}(\rho)$. Then $\pi$ strongly commutes with $\rho$ if and only if the condition $(\star)$ is satisfied.

Proof. Since $\pi$ is integrable, for each $x \in \mathfrak{A}_{h}, \pi(x)$ is essentially selfadjoint. By [7, Corollary 8.2.8 or Chapter 9], $\mathscr{C}^{s}(\pi)^{\prime}$ is generated by the spectral projections of all selfadjoint operators $\overline{\pi(x)}$ with $x \in \mathfrak{A}_{h}$. Therefore $\mathscr{C}^{s}(\pi)^{\prime}$ is generated by all unitary operators $V_{\pi(x)}$ with $x \in \mathfrak{A}_{h}$;

$$
\mathscr{C}^{s}(\pi)^{\prime}=\left\{V_{\pi(x)}: x \in \mathfrak{A}_{h}\right\}^{\prime \prime} \text {. }
$$


Suppose the condition $(\star)$ is satisfied. By Theorem $5,\left\{V_{\pi(x)}: x \in \mathfrak{A}_{h}\right\} \subseteq$ $\mathscr{C}^{w}(\rho)$. By our assumption, $\mathscr{C}^{s}(\rho)$ is a von Neumann algebra with $\mathscr{C}^{s}(\rho)=$ $\mathscr{C}^{w}(\rho)$. Hence $\left\{V_{\pi(x)}: x \in \mathfrak{A}_{h}\right\}^{\prime \prime} \subseteq \mathscr{C}^{s}(\rho)$, so that $\pi$ strongly commutes with $\rho$.

The converse is clear by Corollary 4 .

\section{Pointwise commutativity}

We introduce the notion of pointwise commutativity for representations by the corresponding Cayley transforms, which seems to be reasonable by the preceding arguments.

Definition. Let $\pi$ and $\rho$ be $*$-representations of $\mathfrak{A}$. We say that $\pi$ commutes pointwise with $\rho$, if

$$
V_{\pi(x)} V_{\rho(x)}=V_{\rho(x)} V_{\pi(x)} \quad \text { for all } x \in \mathfrak{A}_{h} .
$$

The following theorem is an improvement of Theorem 6 in [5] in terms of the pointwise commutativity. A representation $\rho$ is said to be an extension of $\pi$ if $\mathscr{D}(\pi) \subseteq \mathscr{D}(\rho)$ and $\pi(a) \eta=\rho(a) \eta$ for all $a \in \mathfrak{A}$ and all $\eta \in \mathscr{D}(\pi)$.

Theorem 7. Let $\pi$ be a closed *-representation of a *-algebra $\mathfrak{A}$ on a Hilbert space $\mathscr{H}$. If $\pi$ has an integrable extension $\rho$ of $\mathscr{H}$ which commutes pointwise with $\pi$, then $\pi=\rho$ and so $\pi$ is itself integrable.

Proof. Suppose $\rho$ is an integrable extension of $\pi$ and commutes pointwise with $\pi$. Take $x$ in $\mathfrak{A}_{h}$. Then $\rho(x)$ is essentially selfadjoint. Let $P$ be the projection of $\mathscr{H}$ onto $\mathscr{R}(\overline{\pi(x)}+i) ; P=V_{\pi(x)}^{*} V_{\pi(x)}$. Since $V_{\rho(x)}$ is unitary, $V_{\rho(x)} V_{\pi(x)}^{*}=V_{\pi(x)}^{*} V_{\rho(x)}$, and so $P$ commutes with $V_{\rho(x)}$. It follows that $P$ commutes with $\overline{\rho(x)}$.

Since $\rho \supseteq \pi$ and $\rho(x)$ is essentially selfadjoint, $\overline{\pi(x)} \subseteq \overline{\rho(x)} \subseteq \pi(x)^{*}$. Hence, for each $\eta \in \mathscr{D}(\overline{\pi(x)})$, we have $P \eta \in P \mathscr{D}(\overline{\rho(x)}) \subseteq \mathscr{D}(\overline{\rho(x)}) \subseteq$ $\mathscr{D}\left(\pi(x)^{*}\right)$ and $P \overline{\pi(x)} \eta=\overline{\rho(x)} P \eta=\pi(x)^{*} P \eta$. Thus $P \overline{\pi(x)} \subseteq \pi(x)^{*} P$. Since $I-P$ is the projection onto $\operatorname{ker}\left(\pi(x)^{*}-i\right)$, we obtain

$$
(I-P) \overline{\pi(x)} \eta=i(I-P) \eta
$$

for each $\eta \in \mathscr{D}(\overline{\pi(x)})$. On the other hand, we have $(I-P) \overline{\pi(x)} \eta=$ $(\overline{\pi(x)}+i P) \eta-P(\overline{\pi(x)}+i) \eta=-i(I-P) \eta$. It follows from the density of $\mathscr{D}(\overline{\pi(x)})$ that $P=I$. Similarly, the projection of $\mathscr{H}$ onto $\mathscr{R}(\overline{\pi(x)}-i)$ is also the identity operator on $\mathscr{H}$. Thus $\pi(x)$ is essentially selfadjoint for each $x \in \mathfrak{A}_{h}$. Therefore $\pi$ is integrable and $\rho=\pi$.

\section{REFERENCES}

1. A. Inoue, H. Kurose, and S. Ôta, Extensions of unbounded representations, Math. Nachr. (to appear).

2. P. E. T. Jørgensen, Operators and representation theory, North-Holland, Amsterdam, 1988.

3. R. V. Kadison and J. R. Ringrose, Fundamentals of the theory of operator algebras, Vol. 1, Academic Press, New York, 1983.

4. G. Lassner, Topological algebras of operators, Rep. Math. Phys. 3 (1972), 279-293.

5. S. Ôta, Commutativity of unbounded representations, preprint, 1991. 
6. R. T. Powers, Self-adjoint algebras of unbounded operators, Comm. Math. Phys. 21 (1971), 85-124.

7. K. Schmüdgen, Unbounded operator algebras and representation theory, Akademie-Verlag, Berlin, 1988.

8. J. Weidmann, Linear operators in Hilbert spaces, Springer-Verlag, New York, 1980.

Department of Mathematics, Kyushu Institute of Design, Fukuoka, 815 Japan 